\title{
Coda: Creativity in psychological research versus in linguistics - Same but different?
}

\author{
Selina Weiss ${ }^{1^{*}}$, Oliver Wilhelm ${ }^{1}$
}

${ }^{1}$ Department of Individual Differences and Psychological Assessment, Institute for Psychology and Education, UIm University, UIm, Baden-Wuerttemberg, Germany

* Corresponding author

E-mail addresses: selina.weiss@uni-ulm.de (S. Weiss)

Author Note:

${ }^{*}$ Corresponding author at: Ulm University, Institute for Psychology and Pedagogy, Selina, Weiss Albert-Einstein Allee 47, 89081 Ulm, Germany. Telephone: +497315031145 . Fax: +49 731/50 31149 .

E-mail: selina.weiss@uni-ulm.de

This is an unedited manuscript that has been accepted for publication in the Special Issue: Construction Grammar and Creativity edited by Thomas

Hoffmann in Cognitive Semiotics. The manuscript will undergo copyediting and typesetting before it is published in its final form. 


\begin{abstract}
:
Understanding the very nature of creativity is a hot topic in research across various disciplines and has profound societal relevance. In this contribution, we discuss verbal creativity by highlighting its definition, psychometric measurement, and relations with other personality dispositions. We relate psychological research with findings from linguistics presented in this issue and depict similarities and differences between both approaches. More specifically, we relate the linguistic terminology of F-creativity to fluency and flexibility, whereas we identify E-creativity as akin to originality. We propose latent semantic analysis as a possible approach for evaluating originality and compare this approach with more commonly applied human ratings. Based on contributions in this issue, we discuss creativity as a domain-general process that is (e.g., in applied arts) often driven by the recombination of mental elements. Lastly, we propose several intelligence and personality dispositions as determinants of individual differences in creativity. We conclude that creativity research in linguistic and psychology has many communalities and interdisciplinary work bears strong promises for the future.
\end{abstract}

Keywords: verbal creativity, domain-specificity, personality, intelligence 


\section{Introduction}

Creativity is the subject of research across disciplines and has resulted in a wide variety of studies and discussions for over a century. While there are numerous debates regarding the nature of creativity, its definition, its measurement, and its relation with other constructs, one thing on which most researchers agree is that creativity is crucial and essential for being successful in life (Gabora and Kaufman 2010). Hennessey and Amabile (2010) describe the great interest in creativity in flourishing research in this area. This can be also exemplified by a quick and simple PsycINFO search (a comprehensive library of research in psychological science): the search term ${ }^{*}$ Creativity produces over 100,000 articles published between 1859 and 2019 and also a large amount of publication outlets for creativity. In particular, the majority of articles were published in the 1980s and 1990s. But just now creativity is in the spotlight again, as large-scale initiatives such as the international educational achievement measure PISA recognize the importance of assessing and fostering creativity (OECD: Vincent-Lancrin 2017). This recent interest is derived from several workforce studies and surveys ( e.g., PWC, 2016) that highlight creativity as the most important human resource in a computerized world. Hence, society and the research community both recognize the significance of defining, understanding, measuring, evaluating, and fostering creativity. Besides, this societal and research interest creativity remains interesting as it is present in everyday life. This general interest is displayed in numerous blogs, books, videos and magazines that tackle creativity.

The numerous studies often only contribute fragments to the understanding of creativity. Therefore, we are in need of a deeper understanding of creativity through more interdisciplinary research (Hennessey and Amabile 2010).

With our contribution to this special issue, we aim to provide an interdisciplinary overview based on psychological research on creativity. Referring to the other contributions, we elaborate on the definition of creativity, criteria for measurability, the discussion regarding the domain specificity of creativity along with the demands of various domains, and the individual differences in creativity while focusing on verbal creativity. 


\section{Definition of creativity}

Creativity research has a long history and some approaches date back to the early $20^{\text {th }}$ century (e.g., rank-ordering the most eminent people in history to analyze their creativity; Cattell 1903). Since then, researchers from different disciplines have attempted to define creativity. Varying definitions and applications of creativity emerged as a product of the given discipline and relevant trends at that time. Some definitions focused on problem solving (Polya 1945) or insight (e.g., Gestalt psychology; Wertheimer 1945), while others focused on specific domains (e.g., creativity in poets; Patrick 1935). Despite the variety of different approaches employed, the core of most definitions applied nowadays is bipartite and includes an original product (new, unusual, novel, unexpected) that is somehow valuable (useful, good, adaptive, appropriate) (Batey 2012; Mumford 2003; Runco and Jaeger 2012). Even though the definition is bipartite, both parts are necessary to describe creativity. Based on this definition, a product cannot be creative without being original and appropriate at the same time. This definition also appears throughout this special issue in several varieties : 1) the interaction of originality/novelty and appropriateness (Hoffmann 2020); 2) the creation of novel form-meaning pairs (Turner 2020); 3) the ability to generate novel work (e.g., creative instances of language use) that is appropriate in the initial context of use (Trousdale 2020); 4) the lack of formal rigidity (oral poetics) that results in a recombination and modification of the formulaic system with a greater focus on flexibility than on novelty (Canovas 2020); 5) the production and understanding of novel output that is constrained by the computational linguistic system (Bergs and Kompa 2020); 6) originality and appropriateness, whereas the latter is not leading to novel ideas in language use, but only the violation of the Norm can result in creativity regarding language (Uhrig 2020). The small variation in these definitions shows that the appropriateness of a new (word-)invention can be in conflict with the language system in question and might thus be difficult to assess. In contrast to appropriateness, we agree that "valuable" (in the sense of pragmatic utility) might be a better fit terminology-wise (Barron 1955). Although a general consensus has been reached regarding this bipartite definition - save some approaches, such as adding surprise as an important character of a novel product (Simonton 2012), for example - the field still lacks a comprehensive and universal definition and a precise operationalization based on this definition (Parkhurst 1999; Plucker et al. 2004). This shortcoming might be seen as intrinsic to the topic: if an overarching and comprehensive definition could be provided and if established measures of it 
existed, then the behavior might not constitute creative acts but something else (for example, inductive generalization).

In addition to these general approaches to define creativity by novelty and value, further efforts to describe more fine-grained creative actions and thoughts can be examined.

The research on creativity in psychology was mainly influenced by Guilford's (1950) presidential address at the American Psychological Association. He described divergent thinking (or divergent production) as a cognitive process that leads to original and novel outcome. According to Guilford (1950), divergent thinking is a pivotal thought process that results in original and valuable outcomes, although it has to mentioned that the terms divergent thinking and creativity cannot be used interchangeably (Runco 2008). This ability to think creatively includes four different dimensions: fluency (quantity of responses), originality (quality of responses), flexibility (variety of responses) and elaboration (number and quality of details provided) (Guilford 1956; Guilford 1960). We think that these dimensions provide a nice approximation of creative thought and action. They can be related with a common definition from linguistics that is featured prominently in this special issue (Bergs and Kompa 2020; Hoffmann 2020): the differentiation between F-creative (fixed and known possibility space) and E-creative (extending or enlarging the existing system) (Sampson 2016). Arguably, F-creativity demonstrates performance in a prescribed space of possible answers. Theoretically, this can be related to fluency and flexibility, as a high performance in both requires the controlled retrieval in a fixed category, as well as switches between given categories (Silvia et al. 2013). Nevertheless, the performance in a flexibility task can lead to an E-creative outcome if the retrieval among existing categories is exceeded. While elaboration can take place in both - a fixed space and in an extended system - originality seems to be theoretically connected to E-creativity. Originality requires not only the retrieval in a given category but also the expansion of the system in order to provide a truly novel and valuable solution. The theoretical link we proposed above requires further empirical validation in an interdisciplinary study. Although F-creative and E-creative are commonly-applied terms in linguistics, it is important to state that true E-creativity might be difficult to conduct in construction grammar, as very creative language applications might be considered as wrong because of the given language norm (Uhrig 2020). Therefore, it is questionable whether someone can be original in construction grammar if it is only defined by truly new and novel outcomes. Likewise, this reality also holds in most other areas where novel interventions are considered as an once-in-a-lifetime creative achievement rather than something that can be em- 
bedded in everyday creative activities. However, construction grammar in particular is bound by several rules that can hinder or restrict the production of original outcomes as they might be considered more incorrect than original. Nonetheless, originality is pivotal and merits consideration. In psychological research, originality is mostly assessed by uniqueness (something unique is unexpected and unusual). Therefore, the question about which objective criteria can be applied to measure creativity in language arises.

\section{Criteria and measurability of creativity in language}

While the definition of creativity is complicated, its operationalization and measurement along with objective criteria of scoring is even more difficult. As stated by Bergs and Kompa (2020), the definition of constraints is crucial to avoid measuring random behavior instead of creativity. In the literature, there are 100 s of tests that can be applied just to measure creativity (Weiss et al. 2020). The literature provides several ways to categorize and describe such measures: a) by defining the object of observation (e.g., the person that is creative, the process of creativity, the environment (press), and the product/outcome; Rhodes 1961), or b) by adding measurement approaches (e.g., self-ratings) and levels (e.g., teams) to the object of observation (Batey 2012). However, these aforementioned heuristic frameworks are not exhaustive and do not allow for a distinct categorization of measurements. All creativity tests can be subsumed in one of the following categories/measurement approaches: test that are self-reports (reports of typical/everyday behavior), reports of others (others' of the target's typical behavior), and ability tests (test data regarding verbal and figural production). Weiss and colleagues (2020) provide an overview of creativity measurement and embed prominent measures in a taxonomy. Self-report measures can either include reports of typical/everyday behavior (e.g., the Epstein Creativity competencies Inventory for Individual's : "I always keep a recording device by my bed at night." [Epstein et al. 2008]) or provide frequencies of creative achievements or actions (e.g., Inventory of Creative Activities and Achievements: "How often have you written a short literary work (e.g., poem, short story) in the past 10 years?" [Diedrich et al. 2018]). Othersreport includes others' (like peers, supervisors, experts, etc.) judgement of typical creative behavior (Scales for Rating the Behavioral Characteristics of Superior Students: "The student demonstrates creative thinking about scientific topics." (Renzulli et al. 2002)) or products, performances, and actions (e.g., the 
Patent Index [Owens et al. 1957]). When assessing verbal creativity, ability tests are the most helpful in terms of reliable and valid measures. Ability tests mostly include open-ended questions with the requirement to verbally (occasionally figurally) provide a creative idea on a given topic (e.g., "Name as many different ways as you can that you can use a brick"). A prominent creativity test is the Torrance Test of Creative Thinking (Torrance 1966). This test includes as most divergent thinking tests do - the actual production of verbal and figural ideas and outcomes. Verbal subtests in the Torrance Test include dimensions such as asking, guessing, and causes while the figural section focuses on picture, construction, and completion. Although the assessment of verbal outcomes or verbally-presented behaviors (as in self-report items) allows for an approximation towards creativity, it remains important to acknowledge that creativity transcends the verbal domain.

Although verbal divergent thinking tests are commonly applied to assess creativity, as in linguistics the question remains what the criterion is to distinguish wrong answers from creative ones: is "cut down a big tree with a brick" as an answer on alternative uses for a brick wrong or creative? The above presented bipartite definition of creativity presents appropriateness/value as a criterion to distinguish wrong from creative. Nevertheless, the appropriateness/value of a product/thought are problematic to judge as the value itself for example is driven by Zeitgeist and culture. Even though variables like Zeitgeist and culture might lead to an underestimation of great ideas and products, the gold standard of judging creativity is with human scorings of originality and appropriateness. The literature provides a huge variety of scoring techniques for such tasks (Reiter-Palmon et al. 2019). One frequently-applied technique is the subjective scoring of answers based on human raters (Silvia et al. 2008). An example for such a scoring is the consensual assessment technique, which is based on experts' rating on a five-point scale that resulted from a consensus of these experts (Amabile 1982; Kaufman et al. 2013). This technique has proven and reliable answers, although it comes with disadvantages. For example, experts might consider different definitions of creativity or might understand/interpret one of its definitions differently (e.g., is novelty defined by a statistical rareness or also by remoteness towards other answers?). Moreover, human scorings are time-consuming and lack true, objective criteria for scoring: if one rater would rate "cutting down a big tree" as wrong while another other would rate it as creative, the raters could either discuss the rating and find a consensus or the ratings would just be averaged.

Linguistic systems provide effective standards and criteria for what can be considered as wrong versus right. So why not learn from these systems and try to find a criteria or method that at least can help us improve the objectivity of 
creativity measurements by serving as an additional standard? A relatively recent objective scoring criteria that seems promising in its application on creativity tasks is the computerized evaluation of open-ended answers based on latent semantic analysis (Landauer et al. 1998). The idea behind these criteria is that creative ideas and hence creative verbal outcomes on these tasks should be remote (Wilson et al. 1953): remote to other answers or remote to the question. This remoteness can be measured by the semantic similarity/distance of answers (Forthmann et al. 2018). Latent semantic analysis has already proven good reliability in tasks that only require a one-word answer (Prabhakaran et al. 2014). Though, as the answer grows longer, the elaboration or amount of details present could bias the results (Forthmann et al. 2018). Besides, such objective scorings lack an evaluation of the value/appropriateness of an answer or may score unique answers as creative even though they are not creative (Silvia et al. 2008). In sum, this score merits further evaluation in a wider range of tasks and different types of answers (e.g., one word versus one sentence versus a short story). Still, we believe that this field and its quite new application on creativity tasks is promising. At this end, the interdisciplinary work of linguists and psychologists can help further elaborate and expand such computerized scoring approaches with the aim of finding more objective criteria to score creative outcomes.

\section{Domain-specificity of creativity}

As described above, most ability tests that measure creativity focus on the verbal output of a person. Nevertheless, the hallmark of creativity research is the ongoing debate about the dimensionality of creativity itself. This debate includes the question about how domain-general or domain-specific skills and creativity are. A domain general skill or trait would indicate that someone who is creative in one area has a higher probability of also being creative in other areas (Silvia et al. 2009). For example, the domain general view would indicate that a person who shows creative accomplishments in visual arts is also a creative writer. Therefore domain-generality should be understood as similarities in the ability of being creative (or not) in various areas. The general definition of creativity implies that a person has original and valuable output in various areas or has novel and appropriate ideas on a variety of topics. Chosen an even more fine-grained aspect of creativity: a person who shows high fluency, high originality, and high flexibility not only in verbal production, but also in figural production. 
Previously, creativity was described as domain-general, including a g-factor view about creativity as a transcending skill or trait (Root-Bernstein 1984). An opposing view discuss creativity as (highly) domain-specific (e.g., domain specificity in educational settings; Barbot et al. 2015; e.g., domain specificity in creative accomplishments; Silvia et al. 2009). This diversity highlights an important question: is creativity domain-specific in the way of broad domains (Baer 1998), task-specific (Baer 1993), or domain-general?

In his contribution, Trousdale (2020) examines to what extent musical and linguistic creativity are similar versus different. He finds similarities such as the adaptation of small items within a larger unit (e.g., improvisation) and differences such as the lack of musical semantics. In sum, he concludes that there are general properties in both systems. Moreover, the argumentation of Trousdale (2020) raises the question whether the differences between creativity in music, language, math, science, etc. are minor: rather, perhaps a general system from that creativity is applied (e.g., recombination of existing patterns). Likewise, Turner (2020) explained that a domain-general process is the basis of creativity in blending. Even in domains that are rather skill-related and require a certain level of expertise (e.g., oral poetics; Canovas 2020), creativity can be achieved by the recombination and reshaping of existing patterns and chunks - as is the case in jazz improvisation (Trousdale 2020). All these contributions show that different domains have varying features in common yet some that are unique. If these domains share enough communalities, they can all be combined under a general factor of creativity. Psychological literature does not provide multivariate studies large enough to prove or counter any view in a statistically-sophisticated way. In sum, we believe that the above-described contributions raises an interesting and relevant point: is there something that remains as a domain-specific component (besides the skill that is necessary to perform creatively in this domain at all) after controlling for general processes such as reorganization and recombination? Probably not.

What might be more applicable in case of domain-specificity is a focus on who is creative in what context. As presented above, measures of previous creative accomplishments include a variety of domains in which someone can be creative, for example literature, music, arts and crafts, creative cooking, or even sports. Moreover, these creative achievements can be further divided into rather great accomplishments for mankind (Big-C; Kaufman and Beghetto 2009) and accomplishments in areas of everyday-creativity (little-c; Richards et al. 1988). They can be even further divided into creative accomplishments in the area of learning and personal experiences (Beghetto and Kaufman 2007; Kaufman and Beghetto 2009). Previous creative accomplishments can be 
measured in all these different areas and all of them might be applicable in linguistics.

\section{Individual differences in verbal creativity}

There is no question that being creative in a specific domain is driven by a level of skill in that domain. If that is the case, then what explanation can account for the fact that everyone is not similarly talented in oral poetics? This artform might indeed appear akin to everyday spoken language, yet only few people can successfully perform this specific type of creative expression. Canovas (2020) offers a first hint on why some people are able while others are not: differences in working memory capacities and retrieval. These differences are referred to as individual differences and are very important to explain the diversity in creative ability and creative outcome. In the following paragraphs, we elaborate on individual differences that have been previously related to creativity and have already been expressed by Hoffmann (2020): intelligence (e.g., Kim 2005) and personality (e.g., McCrae 1987).

\subsection{Intelligence and working memory}

In his contribution to this special issue, Canovas (2020) describes that oral poetics and the general handling of language in patterns requires chunking and therefore involves working memory capacity and retrieval. The nature of oral poetics thus indicates that creativity in language requires a specific amount of working memory. Working Memory Capacity reflects the differences between persons regarding the capacity of their "cognitive system responsible for providing access to information required for ongoing cognitive processes" (Wilhelm et al. $2013 p$. 1). Hence, the capacity of this storage might distinguish very creative oral poets from uncreative ones. In the psychological literature, creativity was often linked - even discussed as being the same - to intelligence. In the $80 \mathrm{~s}$, there were still doubts about the distinction of creativity and general intelligence (Wallach and Kogan 1965). This discussion goes back to the hierarchical integration of creativity in several prominent intelligence models. Creativity can be understood in the framework of the Structure-of-Intellect (SOI) model (Guilford 1966), the Berliner Intelligenzstrukturmodell (Jäger et al. 1997), and the Three- 
Stratum Modell (Carroll 1993). Recent findings support such hierarchical integration and report substantial correlations between broad retrieval ability and creative fluency/originality (Silvia et al. 2013). However, the hierarchy between intelligence and creativity has also been scrutinized lately (Preckel et al. 2011). Meta-analytic findings suggest that intelligence and creativity are only weakly related to one another $(r=.17 ; 95 \% \mathrm{Cl}[.17, .18] ; N=45,880$; Kim 2005). Academic achievement and creativity are also only moderately correlated $(r=.22$; $95 \% \mathrm{Cl}[.19, .24] ; N=52,578$; Gajda et al. 2017). It is important to note that these results might arise due to very different operationalizations and a wide variety in the measurement of creativity (e.g., higher correlations for verbal ability tests with academic achievement $(r=.30)$ than for self-reported creativity with academic achievement $(r=.12)$; Gajda et al. 2017). Such theoretical allocations along with a large body of diverse findings might question the relationship between creativity and intelligence, although Silvia and colleagues (2013) state, based on a thorough multivariate study, that the connection between creativity and intelligence might be more close than previously described in the creativity literature. Working memory shows similar results: it only weakly predicts creativity (Benedek et al. 2014). The sum of these findings indicates that working memory and intelligence are important to be creative and that individual differences in creativity go back to differences in working memory (Canovas 2020) or in knowledge and vocabulary (Bergs and Kompa 2020; Canovas 2020; Hoffmann 2020; Trousdale 2020; Uhrig 2020). However, it also implies that, in order to be creative, we need more than intelligence (knowledge) or working memory and that these are not the same - even though one might require the other, especially in linguistics. Investigations in the so-called threshold theory have shown that there is arguably a nonlinear relation between intelligence and creativity (Holling and Kuhn 2008). There is a great debate between researchers reporting a threshold for creativity: intelligence is proposed as a pre-condition for creativity when under this threshold value, whereas neither are as connected when over the threshold (Jauk et al. 2013). On the other hand, other researchers criticize the various reported thresholds and rather deem intelligence as necessary for creativity (Karwowski et al. 2016).

\subsection{Personality}

Personality traits, like extraversion and openness, have been connected to language acquisition and blending (Hoffmann 2020; Turner 2020). Being open to- 
wards new ideas, values, or fantasies seems to be quite in line with being creative. In the Big Five personality framework, the factor Openness and its underlying traits (such as fantasy, curiosity, and flexibility) are generally linked to creativity (Feist 1998; Furnham and Batey 2006) and especially linked to divergent thinking (verbal creative ability) ( $r=.39$; McCrae 1987). The literature in the past 30 years has consistently report a small to moderate relation between openness and creativity, while other personality traits (such as conscientiousness, neuroticism, and agreeableness) have mostly been unrelated to creativity (Kandler et al. 2016; McCrae 1987; Puryear et al. 2017). In detail, extraversion has sometimes been found to be positively related to divergent thinking (Martindale and Dailey 1996). The factors conscientiousness, neuroticism, and agreeableness are mostly unrelated to creativity (McCrae 1987), even though some findings for example describe artists as more anxious, emotional, and sensitive, while others show that highly-creative scientists are lower in conscientiousness and agreeableness (Feist 1998). Besides, other personality traits, such as psychoticism (Barron and Harrington 1981) or honesty-humility (Silvia et al. 2011), have been previously related to creativity: for example, participants with high creativity values show lower honesty-humility. In sum, we see again that our personality may influence how prone to creativity we are, but it is clearly distinguishable from creativity itself.

\section{General conclusion}

In sum, we have showed that linguistics and psychology share similarities when defining, understanding, measuring, and evaluating creativity. Psychological research and its applications highly rely on verbal production and outcomes as a proxy of creativity. While several problems might arise when using this approach, it also enables us to directly link creativity in psychology and linguistics. At this end, interdisciplinary work can provide a better inside in understanding creativity as a whole as well as measuring creativity (Hennessey and Amabile 2010). Such interdisciplinary studies can be driven by a) unifying terminology in order to prevent jangle fallacies (e.g., (preventing) different terms for the same constructs if they are equivalent; Kelley (1927)) b) finding empirical support for domain-general processes (e.g., can we find processes in psychology that are domain-general, inspired by conceptual blending, that were described as domain-general and necessary for performing E-creativity (Hoffmann 2019)), c) elaborate on objective scorings of verbal creativity (e.g., such as computerized scoring approaches as LSA or construction grammar). Alongside 
all the communalities and differences in the aforementioned areas, we can all agree on the importance of the construct for all disciplines. Although the topic is of such a great interest, there is a long way to go before we can claim to understand creativity.

\section{References}

Amabile, Teresa M. 1982. Social psychology of creativity: A consensual assessment technique. Journal of Personality and Social Psychology 43(5). 997-1013.

Baer, John. 1993. Divergent thinking and creativity: A task-specific approach. Hillsdale, NJ, US: Lawrence Erlbaum Associates, Inc.

Baer, John. 1998. The Case for Domain Specificity of Creativity. Creativity Research Journal 11(2). 173-177.

Barbot, B., Besancon, M., Lubart, T., 2015. Creative potential in educational settings: its nature, measure, and nurture. Education 43(3), 371-381.

Barron, Frank \& David M. Harrington. 1981. Creativity, Intelligence, and Personality. Annual Review of Psychology 32(1). 439-476.

Barron, Frank. 1955. The disposition toward originality. The Journal of Abnormal and Social Psychology 51(3). 478-485.

Batey, Mark. 2012. The Measurement of Creativity: From Definitional Consensus to the Introduction of a New Heuristic Framework. Creativity Research Journal 24(1). 55-65.

Beghetto, Ronald A. \& James C. Kaufman. 2007. Toward a broader conception of creativity: A case for "mini-c" creativity. Psychology of Aesthetics, Creativity, and the Arts 1(2). 73-79.

Benedek, Mathias, Emanuel Jauk, Markus Sommer, Martin Arendasy \& Aljoscha C. Neubauer. 2014. Intelligence, creativity, and cognitive control: The common and differential involvement of executive functions in intelligence and creativity. Intelligence 46. 73-83.

Bergs, Alexander \& Nikola A. Kompa. 2020. Creativity within and outside the linguistic system. Cognitive Semiotics.

Canovas, Cristobal P. 2020. Learning Formulaic Creativity: Chunking in Verbal Art and Speech. Cognitive Semiotics.

Carroll, John. B. 1993. Human cognitive abilities: A survey of factor-analytic studies. New York: Cambridge University Press.

Cattell, James M. 1903. A statistical study of eminent men. Popular Science Monthly 62. 359-377. 
Diedrich, Jennifer, Emanuel Jauk, Paul J. Silvia, Jeffrey M. Gredlein, Aljoscha C. Neubauer \& Mathias Benedek. 2018. Assessment of real-life creativity: The Inventory of Creative Activities and Achievements (ICAA). Psychology of Aesthetics, Creativity, and the Arts 12(3). 304-316.

Epstein, Robert, Steven M. Schmidt \& Regina Warfel. 2008. Measuring and Training Creativity Competencies: Validation of a New Test. Creativity Research Journal 20(1). 7-12.

Feist, Gregory J. 1998. A Meta-Analysis of Personality in Scientific and Artistic Creativity. Personality and Social Psychology Review 2(4). 290-309.

Forthmann, Boris, Oluwatosin Oyebade, Adebusola Ojo, Fritz Günther \& Heinz Holling. 2018. Application of Latent Semantic Analysis to Divergent Thinking is Biased by Elaboration. The Journal of Creative Behavior 53(4). 559-575.

Furnham, Adrian \& Mark Batey. 2006. Creativity, intelligence, and personality: A critical review of the scattered literature. Genetic, Social, and General Psychology Monographs 132(4). 355-429.

Gabora, Liane \& Scott Barry Kaufman. 2010. Evolutionary Approaches to Creativity. In James C Kaufman \& Robert Sternberg (eds.), The Cambridge Handbook of Creativity, 279-300. Cambridge, UK: Cambridge University Press.

Gajda, Aleksandra, Maciej Karwowski \& Ronald A. Beghetto. 2017. Creativity and academic achievement: A meta-analysis. Journal of Educational Psychology 109(2). 269-299.

Guilford, Joy. P. 1966. Intelligence: 1965 model. American Psychologist 21(1). 20-26.

Guilford, Joy P. 1950. Creativity. American Psychologist 5. 444-454.

Guilford, Joy P. 1956. The Structure of Intellect. Psychological Bulletin (53). 267-293.

Guilford, Joy P. 1960. Basic Conceptual Problems in the Psychology of Thinking. Annals of the New York Academy of Sciences 91(1). 6-21.

Hennessey, Beth A. \& Teresa M. Amabile. 2010. Creativity. Annual Review of Psychology 61(1). 569-598.

Hoffmann, Thomas. 2019. Language and creativity: a Construction Grammar approach to linguistic creativity. Linguistics Vanguard 5(1). 1-8.

Hoffmann, Thomas. 2020. Construction Grammar and Creativity: Evolution, psychology and cognitive science. Cognitive Semiotics.

Holling, Heinz \& Jörg-Tobias Kuhn. 2008. Does intellectual giftedness affect the factor structure of divergent thinking? Evidence from a MG-MACS analysis. Psychology Science 50(2). 283-294.

Jäger, A.O., Heinz Martin Süß \& A. Beauducel. 1997. Berliner Intelligenzstruktur-Test: BIS-Test. Göttingen: Hogrefe. 
Jauk, Emanuel, Mathias Benedek, Beate Dunst \& Aljoscha C. Neubauer. 2013. The relationship between intelligence and creativity: New support for the threshold hypothesis by means of empirical breakpoint detection. Intelligence 41(4). 212-221.

Kandler, Christian, Rainer Riemann, Alois Angleitner, Frank M. Spinath, Peter Borkenau \& Lars Penke. 2016. The nature of creativity: The roles of genetic factors, personality traits, cognitive abilities, and environmental sources. Journal of Personality and Social Psychology 111(2). 230-249.

Karwowski, Maciej, Jan Dul, Jacek Gralewski, Emanuel Jauk, Dorota M. Jankowska, Aleksandra Gajda, Michael H. Chruszczewski \& Mathias Benedek. 2016. Is creativity without intelligence possible? A Necessary Condition Analysis. Intelligence 57. 105-117.

Kaufman, James C., John Baer, David H. Cropley, Roni Reiter-Palmon \& Sarah Sinnett. 2013. Furious activity vs. understanding: How much expertise is needed to evaluate creative work? Psychology of Aesthetics, Creativity, and the Arts 7(4). 332-340.

Kaufman, James C. \& Ronald A. Beghetto. 2009. Beyond Big and Little: The Four C Model of Creativity. Review of General Psychology 13(1). 112.

Kelley, Truman L. 1927. Interpretation of Educational Measurments. Yonkers NY: World Book.

Kim, Kyung H. 2005. Can only intelligent people be creative? A meta-analysis. Journal of Secondary Gifted Education 16(2-3). 57-66.

Landauer, Thomas K, Peter W. Foltz \& Darrell Laham. 1998. An introduction to latent semantic analysis. Discourse Processes 25(2-3). 259-284.

Martindale, Colin \& Audrey Dailey. 1996. Creativity, primary process cognition and personality. Personality and Individual Differences 20(4). 409414.

McCrae, Robert R. 1987. Creativity, divergent thinking, and openness to experience. Journal of Personality and Social Psychology 52(6). 12581265.

Mumford, Michael D. 2003. Where Have We Been, Where Are We Going? Taking Stock in Creativity Research. Creativity Research Journal 15(2-3). 107-120.

Owens, William. A., Charles. F. Schumacher \& J. B. Clark. 1957. The measurement of creativity in machine design. Journal of Applied Psychology 41(5). 297-302.

Parkhurst, Howard B. 1999. Confusion, Lack of Consensus, and the Definition of Creativity as a Construct. The Journal of Creative Behavior 33(1). $1-21$.

Patrick, Catharine. 1935. Creative thought in poets. Archives of Psychology 26. 1-74. 
Plucker, Jonathan A., Ronald A. Beghetto \& Gayle T. Dow. 2004. Why Isn't Creativity More Important to Educational Psychologists? Potentials, Pitfalls, and Future Directions in Creativity Research. Educational Psychologist 39(2). 83-96.

Polya, George. 1945. How to solve it: a new aspect of mathematical method (Princeton Science Library). Expanded Princeton Science Library ed. Princeton [N.J.]: Princeton University Press.

Prabhakaran, Ranjani, Adam E. Green \& Jeremy R. Gray. 2014. Thin slices of creativity: Using single-word utterances to assess creative cognition. Behavior Research Methods 46(3). 641-659.

Preckel, Franzis, Christina Wermer \& Frank M. Spinath. 2011. The interrelationship between speeded and unspeeded divergent thinking and reasoning, and the role of mental speed. Intelligence 39(5). 378-388.

Puryear, Jeb S., Todd Kettler \& Anne N. Rinn. 2017. Relating Personality and Creativity: Considering What and How We Measure. The Journal of Creative Behavior 53(2). 232-245.

PWC. 2016. 19th annual global CEO survey. http://www.pwc.com/gx/en/ceosurvey/2016/landing-page/pwc-19th-annual-global-ceo-survey.pdf.

Reiter-Palmon, Roni, Boris Forthmann \& Baptiste Barbot. 2019. Scoring divergent thinking tests: A review and systematic framework. Psychology of Aesthetics, Creativity, and the Arts 13(2). 144-152.

Renzulli, Joseph S, Linda H Smith, Alan J White, Carolyn M Callahan, Robert K Hartman \& Karen L Westberg. 2002. Scales for rating the behavioral characteristics of superior students. Technical and administration manual. Creative Learning Press.

Rhodes, Mel. 1961. An Analysis of Creativity. The Phi Delta Kappan 42. 305310.

Richards, Ruth, Dennis K. Kinney, Maria Benet \& Ann P. Merzel. 1988. Assessing everyday creativity: Characteristics of the Lifetime Creativity Scales and validation with three large samples. Journal of Personality and Social Psychology 54(3). 476.

Root-Bernstein, Robert S. 1984. Creative Process as a Unifying Theme of Human Cultures. Daedalus 113. 197-219.

Runco, Mark A. 2008. Commentary: Divergent thinking is not synonymous with creativity. Psychology of Aesthetics, Creativity, and the Arts 2(2). 93-96.

Runco, Mark A. \& Garrett J. Jaeger. 2012. The Standard Definition of Creativity. Creativity Research Journal 24(1). 92-96.

Sampson, G., 2016. Two ideas of creativity. In: Hinton, M. (Ed.), Evidence, Experiment and Argument in Linguistics and Philosophy of Language. Peter Lang, Bern, pp. 15-26. 
Silvia, Paul J., Roger E. Beaty \& Emily C. Nusbaum. 2013. Verbal fluency and creativity: General and specific contributions of broad retrieval ability $(\mathrm{Gr})$ factors to divergent thinking. Intelligence 41(5). 328-340.

Silvia, Paul J., James C. Kaufman \& Jean E. Pretz. 2009. Is creativity domainspecific? Latent class models of creative accomplishments and creative self-descriptions. Psychology of Aesthetics, Creativity, and the Arts 3. 139-148.

Silvia, Paul J., James C. Kaufman, Roni Reiter-Palmon \& Benjamin Wigert. 2011. Cantankerous creativity: Honesty-Humility, Agreeableness, and the HEXACO structure of creative achievement. Personality and Individual Differences 51(5). 687-689.

Silvia, Paul J., Beate P. Winterstein, John T. Willse, Christopher M. Barona, Joshua T. Cram, Karl I. Hess, Jenna L. Martinez \& Crystal A. Richard. 2008. Assessing creativity with divergent thinking tasks: Exploring the reliability and validity of new subjective scoring methods. Psychology of Aesthetics, Creativity, and the Arts 2(2). 68-85.

Simonton, Dean K. 2012. Taking the U.S. Patent Office Criteria Seriously: A Quantitative Three-Criterion

Creativity Definition and Its Implications. Creativity Research Journal 24(2-3). 97-106.

Torrance, Ellis. P. 1966. The Torrance Tests of Creative Thinking-Norms, Technical Manual Research Edition_Verbal Tests, Forms A and BFigural Tests, Forms $A$ and B. Princeton [N.J.]: Personnel Press.

Trousdale, Graeme. 2020. Creativity, reuse and regularity in music and language. Cognitive Semiotics.

Turner, Mark. 2020. Constructions and Creativity. Cognitive Semiotics.

Uhrig, Peter. 2020. Creative Intentions - The fine line between 'creative' and 'wrong.' Cognitive Semiotics.

Vincent-Lancrin, Stéphan. 2017. Future learning skills in a global context: from discourse to practice. http://docplayer.net/51475061-Futurelearning-skills-in-a-global-context-from-discourse-to-practice.html.

Wallach, Michael A. \& Nathan Kogan. 1965. A new look at the creativity-intelligence distinction1. Journal of Personality 33(3). 348-369.

Weiss, Selina, Oliver Wilhelm \& Patrick Kyllonen. 2020. A Review and Taxonomy of Creativity Measures. Psychology of Aesthetics, Creativity, and the Arts. Submitted for publication.

Wertheimer, M. 1945. Productive Creativity. New York: Harper \& Row.

Wilhelm, Oliver, Andrea Hildebrandt \& Klaus Oberauer. 2013. What is working memory capacity, and how can we measure it? Frontiers in Psychology 4.

Wilson, Robert C., Joy P. Guilford \& Paul R. Christensen. 1953. The measurement of individual differences in originality. Psychological Bulletin 50(5). 362-370. 
Interdisciplinary Creativity Research 